\title{
Citations
}

\section{A Sense of Proportion}

Mass spectrometric determination of the ratio of a probe and its reaction product formed on exposure to hydrogen peroxide $\left(\mathrm{H}_{2} \mathrm{O}_{2}\right)$ looks poised to substantially improve measurement of reactive oxygen species (ROS) in mitochondria of living organisms. ROS such as $\mathrm{H}_{2} \mathrm{O}_{2}$ mediate mitochondrial oxidative damage and redox signaling, and are usually studied using fluorescent probes, limiting analysis to cell culture or easily imaged organisms such as zebrafish and nematodes. The new ratiometric strategy for measuring $\mathrm{H}_{2} \mathrm{O}_{2}$, described in a Cell Metabolism article from Cochemé et al., uses a probe consisting of an arylboronic acid conjugated to a triphenylphosphonium (TPP) cation. This first component forms a phenol group upon reaction with $\mathrm{H}_{2} \mathrm{O}_{2}$, and the TPP moiety harnesses membrane potential to accumulate in the mitochondrial matrix. The probe in its boronic acid form (MitoB) can be easily distinguished from the phenol-containing product (MitoP) by mass spectrometry. In a typical experiment in Drosophila (the model system used in the article), flies are given an intrathoracic injection of MitoB, then a whole-fly homogenate is prepared. After spiking with an internal standard and sample preparation, analysis occurs by liquid chromatography-tandem mass spectrometry, followed by preparation of a standard curve, quantitation, and calculation of the MitoP/MitoB ratio. The authors present several lines of evidence that this ratio reflects mitochondrial $\mathrm{H}_{2} \mathrm{O}_{2}$ production. However, they do note that this method gives an average $\mathrm{H}_{2} \mathrm{O}_{2}$ measurement, so it might miss local fluctuations. Even so, the technique is the first to allow prediction of absolute $\mathrm{H}_{2} \mathrm{O}_{2}$ concentration. Furthermore, initial tests showed MitoB can also be used in nematodes and mice (where it appears that the MitoP/MitoB ratio can be quantified from urine). In cell culture, MitoP equilibrates with culture medium, opening the possibility for repeated $\mathrm{H}_{2} \mathrm{O}_{2}$ sampling. This methodology, which can be extended to other ROS, should be relevant to research on aging and physiological responses to oxidative damage.

Cochemé et al. 2011. Measurement of $\mathrm{H}_{2} \mathrm{O}_{2}$ within living Drosophila during aging using a ratiometric mass spectrometry probe targeted to the mitochond rial matrix. Cell Metab. 13:340-350.

\section{The Strong, Silent Type}

Seeking potent short hairpin RNAs (shRNAs) for gene silencing can be disheartening. In theory, shRNAs make for a desirable alternative to small interfering RNA (siRNA) duplexes because they can be expressed from polymerase II promoters in the context of microRNA (miRNA) transcripts, avoiding the need for transient transfection of exogenous siRNAs. However, a critical disadvantage of shRNAs is the lack of an algorithm that can reliably predict which sequences will lead to the greatest degree of silencing. Experience shows that siRNA-directed algorithms do not predict effective shRNAs well. Instead, candidate shRNAs must be empirically tested to find out which are potent enough to be effective when expressed from a single integrated copy of the expression cassette-a key criterion for functional genomics screens, and a useful way to prevent the nonspecific toxicity that can happen when shRNAs are overexpressed. In an article published in Molecular Cell, Fellmann et al. describe a screening approach for rare potent shRNAs. Their readout is pSENSOR, a plasmid that expresses both the shRNA (in a regulatable manner) and its target (in the $3^{\prime}$ untranslated region of a Venus-encoding transcript). In this system, doxycycline induction of shRNA expression decreases Venus fluorescence to a degree proportional to the potency

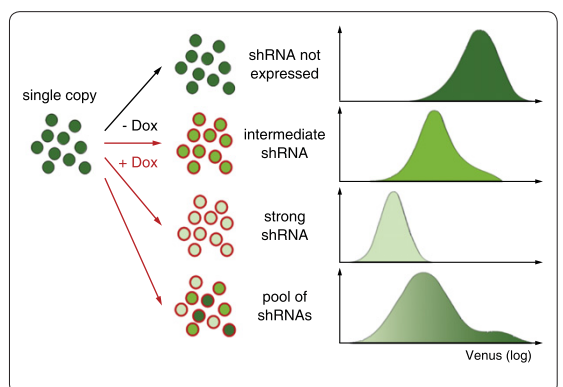

Flow cytometric screening for potent shRNAs in retroviral vector-transduced cells. Reproduced with permission (c) 2011 CELL PRESS.

of the shRNA. The authors constructed a library of approximately 20,000 pSENSORs by on-chip synthesis of oligonucleotides that each contain an shRNA and its target sequence. Subsequent cloning steps produced a murine retroviral vector expressing a given shRNA in an miRNA context followed by a Venus expression cassette with the cognate target sequence. This pSENSOR library, which reflects all possible shRNAs for nine mammalian transcripts, was then transduced into an avian cell line (to minimize shRNA silencing of endogenous transcripts) and potent shRNAs were selected with successive cycles of cell sorting by flow cytometry. After running just four cycles and scoring the "winners" according to their enrichment factor and whether they were pulled out of replicate assays, $85-90 \%$ of the resulting shRNAs showed strong silencing when expressed from a single integrated provirus. Tellingly, $70 \%$ of the successful shRNAs were not listed in the top 50 suggestions of three siRNA design algorithms. Moreover, the sequence rules the authors were able to infer from their screen results differed from known design constraints of siRNAs. The results, together with the observation that on average only $2.4 \%$ of possible shRNAs are potent silencers, emphasize the value of performing shRNA screens. Researchers designing shRNAs can use these newly discovered rules to prepare a simplified library of shRNA candidates, and then use the pSENSOR screen to hone in on the most potent silencers.

Fellmann et al. Functional identification of optimized RNAi triggers using a massively parallel sensor assay. Mol Cell. 41:733-746.

Selected and written by Nijsje Dorman, Ph.D. 四

BioTechniques 50:279 (May 2011)

doi 10.2144/000113661

To purchase reprints of this article, contact:

biotechniques@fosterprinting.com 